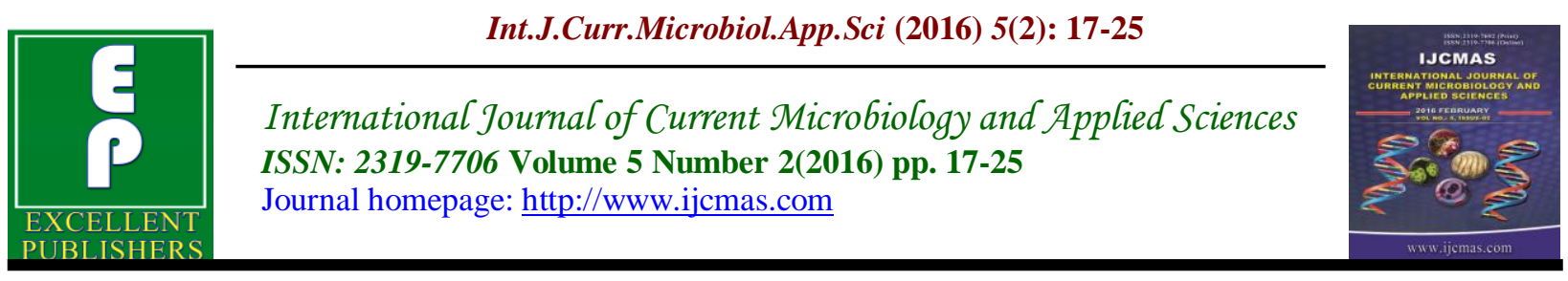

Original Research Article

http://dx.doi.org/10.20546/ijcmas.2016.502.002

\title{
Effect of Integrated Weed Management Practices on Growth and Yield of Bt-Cotton in Telangana State, India
}

\author{
P. Leela Rani ${ }^{1 *}$, M. Yakadri and T. Ramprakash \\ AICRP on Weed Management, College of Agriculture, Rajendra Nagar, Hyderabad, India \\ *Corresponding author
}

\begin{abstract}
A B S T R A C T
Keywords

IWM,

Bt cotton,

Economics,

Weed

Management

\section{Article Info}

Accepted:

09 Januaryr 2016

Available Online:

10, February 2016

The field experiment was carried out at college farm, Professor Jayashankar Telangana State Agricultural University, Rajendranagar, Hyderabad during kharif, 2014 with ten weed management practices with an object to find out the most effective weed management practice in Bt cotton. Uncontrolled weed growth during crop growing season resulted in yield loss of upto $86 \%$. Increased yield of 62.6 to $85.9 \%$ was observed with various weed management practices. More number of boles per plant (23) kapas yield plant ${ }^{-1}\left(92 \mathrm{~g}\right.$ plant $\left.^{-1}\right)$ and kapas yield (1427 kg ha-1) were noticed with mechanical weeding thrice at 20, 40 and 60 DAS and was significantly differed from all other treatments under study, but it was followed by pre emergence application of pendimethalin @ $1000 \mathrm{~g} \mathrm{ha}^{-1}$ followed by 2 hand weedings at 20 and 50 DAS, inturn and was onpar with pre emergence application of early post emergence application of pyrithiobac-sodium + quizalofop- p- ethyl @ 62.5+50 $\mathrm{g} \mathrm{ha}^{-1} \mathrm{fb}$ manual weeding at 50 DAS. Higher gross returns (Rs 53513), net retuns (Rs 26763) and B.C ratio (2.0) also obtained from mechanical weeding thrice at 20,40 and 60 DAS with lower weed drymatter, weed density and higher WCE.
\end{abstract}

\section{Introduction}

The use of Bt-Cotton in India has grown exponentially since its introduction. Cotton being a wide spaced and relatively slow growing crop during its initial growth stages, suffers from severe weed competition and causing substantial reduction in seed cotton yields upto an extent of 69 per cent (Srinivasulu and Rao, 2000). Weed species in cotton field differ widely due to soil and environmental conditions. Weed control, especially during the first eight weeks of cotton growth is essential due to the vulnerability of cotton to early season weed competition (Buchanan and Burns, 1970). Panwar and Malik (1991) reported that the competition of Trianthema portulacastrum was higher up to 50 DAS, whereas, competition of Echinochloa crusgalli was up to 100 DAS. Hence, first 60 DAS was the most critical period for cropweed competition.

So, use of herbicides is one of the best options to avoid the competition from weeds 
during the critical period of crop growth. Most often due to incessant rains during kharif season, hand weeding and intercultivation (IC) become difficult in cotton. Further, labours being scarce and costly, growers are forced to fall back on chemicals for weed control. Pre emergence herbicides at recommended doses are generally capable of controlling annual weeds upto a period of 30 days (Pawar et al., 2000). Concentration of these herbicides in soil decreases due to the short half life of herbicide molecules leading to emergence of susceptible weed species beyond 30 days after application of herbicides. In the absence of interculture and with regular monsoon rains, weeds germinate in different spells and compete with crop plants and finally reduce the seed cotton yield. Hence, there is a need to go for sequential application of PRE followed by POE herbicides to manage the late emerging weeds to eliminate weed competition throughout the critical period (Pawar et al., 2000). the productivity of seed cotton in India is $537 \mathrm{~kg} \mathrm{ha}^{-1}$ which is below the world average of $790 \mathrm{~kg} \mathrm{ha}^{-1}$. In Telangana, the cotton crop is being grown in an area of 16.51 lakh ha with the productivity of 515 $\mathrm{kg} \mathrm{ha}^{-1}$. This crop is mostly grown in alfisols of Southern Telangana agro climatic zone. Hence, present investigation was conducted to study the economics of irrigated $B t$ cotton as influenced by sequential application of herbicides.

\section{Materials and Methods}

The field experiment was carried out at college farm, Professor Jayashankar Telangana State Agricultural university, Rajendranagar, Hyderabad situated at an altitude of $542.3 \mathrm{~m}$ above mean sea level at $17^{\circ} 19^{\prime} \mathrm{N}$ latitude and $78^{\circ} 23^{\prime} \mathrm{E}$ longitude during kharif, 2014. The experiment was laid out in a randomized block design with 3 replications and 10 treatments viz., T1,
Pendimethalin@ @ $1000 \mathrm{fb} 2 \mathrm{HW}$ at 20 and 50 DAS: $\mathrm{T}_{2}$, Pendimethalin @ $1000 \mathrm{fb}$ pyrithiobac-sodium @ $62.5 \mathrm{~g} \mathrm{ha}{ }^{-1}: \mathrm{T}_{3}$, Pendimethalin @ $1000 \mathrm{fb}$ pyrithiobacsodium@62.5 $\mathrm{g} \mathrm{ha}^{-1}$ + quizalofop- p- ethyl @ $50.0 \mathrm{~g} \mathrm{ha}^{-1}: \mathrm{T}_{4}$, pyrithiobac-sodium @ $62.5 \mathrm{~g} \mathrm{ha}^{-1}+$ quizalofop- p- ethyl @ $50.0 \mathrm{~g}$ ha ${ }^{-1}: \mathrm{T}_{5}$ pyrithiobac-sodium @ $62.5 \mathrm{~g} \mathrm{ha}^{-1}+$ quizalofop- p- ethyl @ $50.0 \mathrm{~g} \mathrm{ha}^{-1} \mathrm{fb}$ manual weeding at 50 DAS; $\mathrm{T}_{6}$ Pyrithiobac -sodium @6 $62.5 \mathrm{~g} \mathrm{ha}^{-1}+$ quizalofop -p- ethyl @ 50.0 $\mathrm{g} \mathrm{ha}^{-1} \mathrm{fb}$ directed spray of paraquat @600 g ha $^{-1} ; \mathrm{T}_{7}$ Pyrithiobac-sodium @ $62.5 \mathrm{~g} \mathrm{ha}^{-1}+$ quizalofop -p- ethyl @ $50.0 \mathrm{~g} \mathrm{ha}^{-1} \mathrm{fb}$ directed spray of glyphosate @ $2000 \mathrm{~g} \mathrm{ha}^{-1}$; $\mathrm{T}_{8}$ Pendimethalin @ $1000 \mathrm{~g} \mathrm{ha}^{-1} \mathrm{fb}$ glyphosate @ $2000 \mathrm{~g} \mathrm{ha}^{-1}$ directed spray; $\mathrm{T}_{9}$ Mechanical weeding thrice at 20, 40 and 60 DAS and $\mathrm{T}_{10}$ weedy check.

The crop was sown on $7^{\text {th }}$ July 2014 with spacing of $90 \mathrm{~cm} \times 60 \mathrm{~cm}$. Fertilizers were applied during crop growing season as per the recommendation (150-60-60 Kg N, $\mathrm{P}_{2} \mathrm{O}_{5}$ and $\mathrm{K}_{2} \mathrm{O}$ ). Herbicides were applied using a knapsack sprayer fitted with flat fan nozzle. Weed count was recorded species wise at 30, 60, 90 and 120 DAS using $0.25 \mathrm{~m}^{2}$ quadrat from each plot and expressed per $\mathrm{m}^{2}$. Five plants were randomly selected in each plot and tagged to record the regular observations. Four pickings of seed cotton yield was taken from each treatment for recording final yield data.

\section{Results and Discussion}

\section{Weed Flora}

Cotton crop was infested with a large number of weeds owing to longer duration, slow initial growth. The important monocotyledonous weeds observed in the experiment during crop growing season were Cyperus rotundus, Cynodondactylon, Dactylactenium aegyptium, Digera muricata, Digiteria sanguinalis, Dinebra 
retroflexa, Echinicloa colona, Eragrostis cilianensis, Panicum spp. While common dicotyledonous weeds observed were Acalypha rhomboidea, Amaranthus polygamus, Cleome viscose, Commelina bhenghalensis, Parthenium hysterophorus and Trianthema portulacastrum.

Table.1 Effect of Different Pre and Post Emergence Herbicides on Weed Dry Matter and Weed Control Efficiency in Cotton During Kharif -2014

\begin{tabular}{|c|c|c|c|c|c|c|c|c|c|}
\hline \multicolumn{2}{|c|}{ Treatment } & \multicolumn{4}{|c|}{ WDM $\left(\mathrm{g} / \mathrm{m}^{2}\right)$} & \multicolumn{4}{|c|}{$\begin{array}{c}\text { Weed Control efficiency } \\
(\%)\end{array}$} \\
\hline & & $\begin{array}{c}30 \\
\text { DAS } \\
\end{array}$ & $\begin{array}{c}60 \\
\text { DAS } \\
\end{array}$ & $\begin{array}{c}90 \\
\text { DAS } \\
\end{array}$ & $\begin{array}{c}120 \\
\text { DAS }\end{array}$ & $\begin{array}{c}30 \\
\text { DAS } \\
\end{array}$ & $\begin{array}{c}\text { 60 } \\
\text { DAS }\end{array}$ & $\begin{array}{c}90 \\
\text { DAS }\end{array}$ & $\begin{array}{l}120 \\
\text { DAS }\end{array}$ \\
\hline $\mathrm{T}_{1}$ & Pendimethalin $\mathrm{fb} 2 \mathrm{HW}$ & $\begin{array}{l}5.33 \\
(28)\end{array}$ & $\begin{array}{l}5.33 \\
(29)\end{array}$ & $\begin{array}{l}9.27 \\
(85)\end{array}$ & $\begin{array}{l}12.63 \\
(161)\end{array}$ & 89.27 & 91.37 & 75.4 & 49.79 \\
\hline $\mathrm{T}_{2}$ & $\begin{array}{l}\text { Pendimethalin } f b \\
\text { pyrithiobac-sodium }\end{array}$ & $\begin{array}{l}11.97 \\
(143)\end{array}$ & $\begin{array}{l}12.79 \\
(163)\end{array}$ & $\begin{array}{l}13.64 \\
(185) \\
\end{array}$ & $\begin{array}{l}13.78 \\
(189) \\
\end{array}$ & 45.34 & 50.80 & 46.5 & 41.07 \\
\hline $\mathrm{T}_{3}$ & $\begin{array}{l}\text { Pendimethalin } f b \\
\text { pyrithiobac-sodium + } \\
\text { quizalofop- p- ethyl }\end{array}$ & $\begin{array}{l}9.95 \\
(103)\end{array}$ & $\begin{array}{l}11.34 \\
(128)\end{array}$ & $\begin{array}{l}12.38 \\
(153)\end{array}$ & $\begin{array}{l}14.25 \\
(207)\end{array}$ & 60.66 & 61.45 & 55.8 & 35.47 \\
\hline $\mathrm{T} 4$ & $\begin{array}{l}\text { Pyrithiobac-sodium + } \\
\text { quizalofop- } \mathrm{p} \text {-ethyl }\end{array}$ & $\begin{array}{l}12.87 \\
(165)\end{array}$ & $\begin{array}{l}14.27 \\
(203)\end{array}$ & $\begin{array}{l}16.17 \\
(261)\end{array}$ & $\begin{array}{l}16.76 \\
(280)\end{array}$ & 36.78 & 38.76 & 24.8 & 12.85 \\
\hline $\mathrm{T}_{5}$ & $\begin{array}{l}\text { Pyrithiobac-sodium }+ \\
\text { quizalofop- p- ethyl } f b \\
\text { manual weeding }\end{array}$ & $\begin{array}{l}11.19 \\
(126)\end{array}$ & $\begin{array}{l}5.49 \\
(29)\end{array}$ & $\begin{array}{l}9.51 \\
(90)\end{array}$ & $\begin{array}{l}12.99 \\
(168)\end{array}$ & 51.72 & 91.16 & 74.0 & 47.71 \\
\hline $\mathrm{T}_{6}$ & $\begin{array}{l}\text { Pyrithiobac -sodium }+ \\
\text { quizalofop -p- ethyl } f b \\
\text { directed spray of paraquat }\end{array}$ & $\begin{array}{l}12.30 \\
(151)\end{array}$ & $\begin{array}{l}12.67 \\
(161)\end{array}$ & $\begin{array}{l}11.14 \\
(124)\end{array}$ & $\begin{array}{l}11.77 \\
(139)\end{array}$ & 42.02 & 51.61 & 64.2 & 56.84 \\
\hline $\mathrm{T}_{7}$ & $\begin{array}{l}\text { Pyrithiobac-sodium }+ \\
\text { quizalofop - p- ethyl } f b \\
\text { directed spray of } \\
\text { glyphosate }\end{array}$ & $\begin{array}{l}11.88 \\
(141)\end{array}$ & $\begin{array}{l}13.29 \\
(176)\end{array}$ & $\begin{array}{c}10.64 \\
(13)\end{array}$ & $\begin{array}{l}11.41 \\
(132)\end{array}$ & 45.85 & 46.99 & 67.3 & 58.92 \\
\hline $\mathrm{T}_{8}$ & $\begin{array}{l}\text { Pendimethalin } f b \\
\text { glyphosate directed spray }\end{array}$ & $\begin{array}{l}10.85 \\
(127)\end{array}$ & $\begin{array}{l}6.38 \\
(40) \\
\end{array}$ & $\begin{array}{l}9.94 \\
(98) \\
\end{array}$ & $\begin{array}{l}11.41 \\
(129) \\
\end{array}$ & 51.47 & 87.95 & 71.7 & 59.75 \\
\hline $\mathrm{T}_{9}$ & Mechanical weeding (3) & $\begin{array}{l}5.14 \\
(127)\end{array}$ & $\begin{array}{l}5.36 \\
(28)\end{array}$ & $\begin{array}{l}6.11 \\
(39) \\
\end{array}$ & $\begin{array}{l}9.57 \\
(91) \\
\end{array}$ & 89.73 & 91.65 & 88.8 & 71.78 \\
\hline $\mathrm{T}_{10}$ & Weedy check & $\begin{array}{l}15.71 \\
(261)\end{array}$ & $\begin{array}{l}18.23 \\
(332)\end{array}$ & $\begin{array}{l}18.77 \\
(352)\end{array}$ & $\begin{array}{l}18.66 \\
(349)\end{array}$ & 0.00 & 0.00 & 0.00 & 0.00 \\
\hline & C.D $(0.05)$ & 4.023 & 1.67 & 1.96 & 2.71 & & & & \\
\hline & C.V & 21.73 & 8.1 & 9.94 & 12.12 & & & & \\
\hline
\end{tabular}


Table.2 Effect of Different Pre and Post Emergence Herbicides on Weed Density and Relativ Density of Cotton during Kharif -2014

\begin{tabular}{|c|c|c|c|c|c|c|c|c|c|}
\hline \multicolumn{2}{|c|}{ Treatment } & \multicolumn{4}{|c|}{ Weed Density $\left(\mathrm{No} / \mathrm{m}^{2}\right)$} & \multicolumn{4}{|c|}{ Relativ Density (\%) } \\
\hline & & 30 & 60 & 90 & 120 & 30 & 60 & 90 & 120 \\
\hline $\mathrm{T}_{1}$ & Pendimethalin $\mathrm{fb} 2 \mathrm{HW}$ & $\begin{array}{c}12.85 \\
165 \\
\end{array}$ & $\begin{array}{l}14.44 \\
(208)\end{array}$ & $\begin{array}{l}11.30 \\
(127)\end{array}$ & $\begin{array}{l}9.80 \\
(97)\end{array}$ & 4.77 & 8.33 & 8.62 & 8.67 \\
\hline $\mathrm{T}_{2}$ & $\begin{array}{l}\text { Pendimethalin } f b \\
\text { pyrithiobac-sodium }\end{array}$ & $\begin{array}{l}17.27 \\
(305)\end{array}$ & $\begin{array}{l}17.03 \\
(292)\end{array}$ & $\begin{array}{l}13.09 \\
(172)\end{array}$ & $\begin{array}{l}10.18 \\
(105)\end{array}$ & 8.81 & 11.69 & 11.65 & 9.38 \\
\hline $\mathrm{T}_{3}$ & $\begin{array}{l}\text { Pendimethalin } f b \\
\text { pyrithiobac-sodium }+ \\
\text { quizalofop- p- ethyl }\end{array}$ & $\begin{array}{l}16.20 \\
(263)\end{array}$ & $\begin{array}{l}16.83 \\
(283)\end{array}$ & $\begin{array}{l}12.32 \\
(155)\end{array}$ & $\begin{array}{l}10.17 \\
(103)\end{array}$ & 7.58 & 11.32 & 10.47 & 9.14 \\
\hline $\mathrm{T} 4$ & $\begin{array}{l}\text { Pyrithiobac-sodium + } \\
\text { quizalofop- } p \text {-ethyl }\end{array}$ & $\begin{array}{l}20.53 \\
(427)\end{array}$ & $\begin{array}{l}15.29 \\
(236)\end{array}$ & $\begin{array}{l}13.16 \\
(176)\end{array}$ & $\begin{array}{l}10.63 \\
(112)\end{array}$ & 12.31 & 9.45 & 11.92 & 9.97 \\
\hline $\mathrm{T}_{5}$ & $\begin{array}{l}\text { Pyrithiobac-sodium }+ \\
\text { quizalofop- p- ethyl } f b \\
\text { manual weeding }\end{array}$ & $\begin{array}{l}22.26 \\
(495)\end{array}$ & $\begin{array}{c}187 \\
13.57\end{array}$ & $\begin{array}{c}149 \\
12.07\end{array}$ & $\begin{array}{c}129 \\
11.36\end{array}$ & 14.28 & 7.48 & 10.11 & 11.52 \\
\hline $\mathrm{T}_{6}$ & $\begin{array}{l}\text { Pyrithiobac -sodium }+ \\
\text { quizalofop }-\mathrm{p} \text { - ethyl } \mathrm{fb} \\
\text { directed spray of paraquat }\end{array}$ & $\begin{array}{l}19.88 \\
(396)\end{array}$ & $\begin{array}{l}15.59 \\
(243)\end{array}$ & $\begin{array}{l}13.18 \\
(176)\end{array}$ & $\begin{array}{l}11.80 \\
(139)\end{array}$ & 11.43 & 9.72 & 11.92 & 12.35 \\
\hline $\mathrm{T}_{7}$ & $\begin{array}{l}\text { Pyrithiobac-sodium }+ \\
\text { quizalofop - p- ethyl } f b \\
\text { directed spray of } \\
\text { glyphosate }\end{array}$ & $\begin{array}{l}19.95 \\
(400)\end{array}$ & $\begin{array}{l}15.85 \\
(252)\end{array}$ & $\begin{array}{l}11.63 \\
(135)\end{array}$ & $\begin{array}{l}10.63 \\
(112)\end{array}$ & 11.54 & 10.09 & 9.12 & 9.97 \\
\hline $\mathrm{T}_{8}$ & $\begin{array}{l}\text { Pendimethalin } f b \\
\text { glyphosate directed spray }\end{array}$ & $\begin{array}{l}16.90 \\
(288)\end{array}$ & $\begin{array}{l}12.52 \\
(157)\end{array}$ & $\begin{array}{l}11.61 \\
(136)\end{array}$ & $\begin{array}{l}11.46 \\
(131)\end{array}$ & 8.31 & 6.30 & 9.21 & 11.64 \\
\hline $\mathrm{T}_{9}$ & Mechanical weeding (3) & $\begin{array}{l}11.31 \\
(128) \\
\end{array}$ & $\begin{array}{l}14.91 \\
(222)\end{array}$ & $\begin{array}{l}8.05 \\
(65)\end{array}$ & $\begin{array}{l}7.22 \\
(53)\end{array}$ & 3.69 & 8.88 & 4.42 & 4.75 \\
\hline $\mathrm{T}_{10}$ & Weedycheck & $\begin{array}{l}24.46 \\
(599)\end{array}$ & $\begin{array}{l}17.97 \\
(323)\end{array}$ & $\begin{array}{l}13.64 \\
(1850\end{array}$ & $\begin{array}{l}11.83 \\
(141)\end{array}$ & 17.28 & 12.92 & 12.55 & 12.59 \\
\hline & C.D $(0.05)$ & 3.51 & 2.63 & 2.96 & 2.22 & & & & \\
\hline & C.V & 11.17 & 9.89 & 14.28 & 12.24 & & & & \\
\hline
\end{tabular}


Table.3 Effect of Different Pre and Post Emergence Herbicides on Growth Parameters of Cotton during Kharif -2014

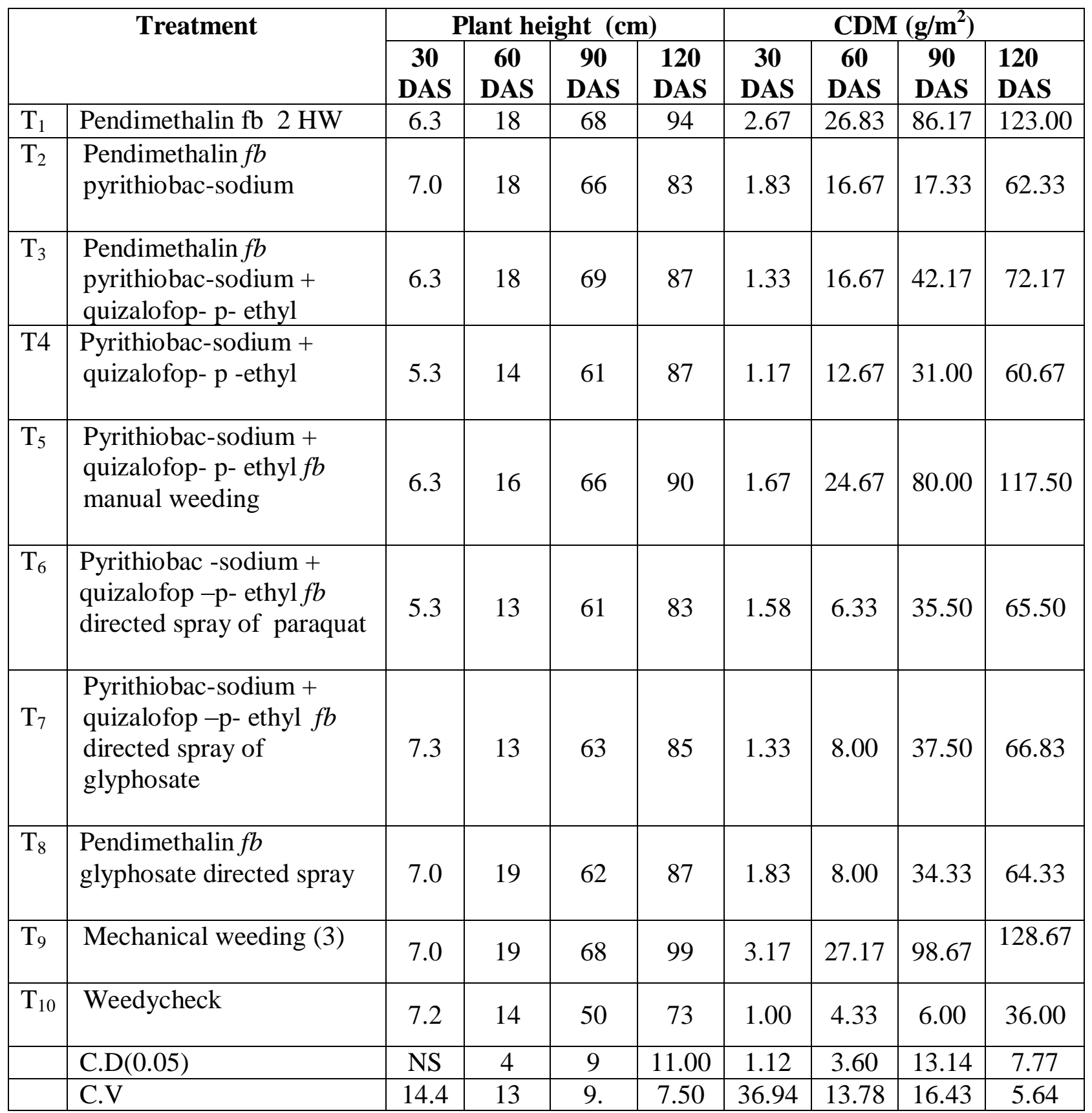


Table.4 Effect of Different Pre and Post Emergence Herbicides on Yield, Yield Attributing and Economics of Cotton during Kharif -2014

\begin{tabular}{|c|c|c|c|c|c|c|c|c|c|}
\hline \multicolumn{2}{|c|}{ Treatment } & \multirow{2}{*}{$\begin{array}{c}\begin{array}{c}\text { No. of } \\
\text { boles/ } \\
\text { plant }\end{array} \\
19\end{array}$} & \multirow{2}{*}{$\begin{array}{c}\begin{array}{c}\text { Kapas } \\
\text { yield } \\
\text { /plant }\end{array} \\
80\end{array}$} & \multirow{2}{*}{$\begin{array}{c}\begin{array}{c}\text { Kapas } \\
\text { yield } \\
\text { kg /ha }\end{array} \\
1209\end{array}$} & \multirow{2}{*}{$\begin{array}{r}\text { Weed } \\
\text { index }\end{array}$} & \multirow{2}{*}{$\begin{array}{c}\begin{array}{c}\text { CC } \\
\text { Rs/ha }\end{array} \\
32840\end{array}$} & \multirow{2}{*}{$\begin{array}{c}\begin{array}{c}\text { GR } \\
\text { Rs/ha }\end{array} \\
45375\end{array}$} & \multirow{2}{*}{$\begin{array}{c}\begin{array}{c}\text { NR } \\
\text { Rs/ha }\end{array} \\
12535\end{array}$} & \multirow{2}{*}{$\begin{array}{l}\text { BC } \\
1.38\end{array}$} \\
\hline $\mathrm{T}_{1}$ & Pendimethalin fb $2 \mathrm{HW}$ & & & & & & & & \\
\hline $\mathrm{T}_{2}$ & $\begin{array}{l}\text { Pendimethalin } f b \\
\text { pyrithiobac-sodium }\end{array}$ & 8 & 44 & 535 & 62.52 & 27127 & 2006 & -25121 & 0.07 \\
\hline $\mathrm{T}_{3}$ & $\begin{array}{l}\text { Pendimethalin } f b \\
\text { pyrithiobac-sodium + } \\
\text { quizalofop- p- ethyl }\end{array}$ & 10 & 49 & 637 & 55.33 & 27449 & 2389 & -25060 & 0.09 \\
\hline $\mathrm{T} 4$ & $\begin{array}{l}\text { Pyrithiobac-sodium + } \\
\text { quizalofop- } p \text {-ethyl }\end{array}$ & 9 & 46 & 583 & 59.14 & 25858 & 2194 & -23664 & 0.08 \\
\hline $\mathrm{T}_{5}$ & $\begin{array}{l}\text { Pyrithiobac-sodium }+ \\
\text { quizalofop- p- ethyl } f b \\
\text { manual weeding }\end{array}$ & 15 & 70 & 1019 & 28.63 & 29608 & 38025 & 8417 & 1.28 \\
\hline $\mathrm{T}_{6}$ & $\begin{array}{l}\text { Pyrithiobac -sodium }+ \\
\text { quizalofop -p- ethyl } f b \\
\text { directed spray of } \\
\text { paraquat }\end{array}$ & 11 & 57 & 783 & 45.10 & 26758 & 2936 & -23822 & 0.11 \\
\hline $\mathrm{T}_{7}$ & $\begin{array}{l}\text { Pyrithiobac-sodium }+ \\
\text { quizalofop -p- ethyl } f b \\
\text { directed spray of } \\
\text { glyphosate }\end{array}$ & 12 & 59 & 806 & 43.53 & 27809 & 3240 & -24569 & 0.12 \\
\hline $\mathrm{T}_{8}$ & $\begin{array}{l}\text { Pendimethalin } f b \\
\text { glyphosate directed spray }\end{array}$ & 13 & 60 & 828 & 41.95 & 27291 & 2925 & -24366 & 0.11 \\
\hline $\mathrm{T}_{9}$ & Mechanical weeding (3) & 23 & 92 & 1427 & 0.01 & 26750 & 53513 & 26763 & 2.00 \\
\hline $\mathrm{T}_{10}$ & Weedycheck & 4 & 26 & 200 & 86.00 & 23750 & 750 & -23000 & 0.03 \\
\hline & C.D $(0.05)$ & 3.63 & 12.48 & 231.34 & & & & & \\
\hline & C.V & 16.96 & 12.4 & 16.671 & & & & & \\
\hline
\end{tabular}

\section{CC: Cost of Cultivation}

GR : Gross Returns

NR : Net Returns

Significantly lower weed drymatter and higher weed control efficiency was observed with mechanical weeding thrice at 20,40 and 60 DAS at all the growth stages (Table $1)$, however it was onpar with pre
Price / kg : Rs 37.50

emergence application of pendimethalin @ $1000 \mathrm{~g} \mathrm{ha}^{-1} \mathrm{fb} 2 \mathrm{HW}$ at 20 and 50 DAS at 30 and 60 DAS and early post emergence application of pyrithiobac-sodium @62.5 g ha $^{-1}+$ quizalofop- p- ethyl @ $50.0 \mathrm{~g} \mathrm{ha}^{-1} \mathrm{fb}$ 
manual weeding at 50 DAS and pre emergence application of pendimethalin @ $1000 \mathrm{~g} \mathrm{ha}^{-1} \mathrm{fb}$ pyrithiobac-sodium @ $62.5 \mathrm{~g}$ ha $^{-1}$ at 60 DAS but significantly superior over all other treatments at 90 DAS. However at 120 DAS it was onpar with early postemergence application of pyrithiobac -sodium @ $62.5 \mathrm{~g} \mathrm{ha}^{-1}+$ quizalofop -p- ethyl @ $50.0 \mathrm{~g} \mathrm{ha}^{-1} \mathrm{fb}$ directed spray of paraquat @600 g ha ${ }^{-1}$, Pyrithiobac -sodium @ $62.5 \mathrm{~g} \mathrm{ha}^{-1}+$ quizalofop -p- ethyl @ $50.0 \mathrm{~g} \mathrm{ha}^{-1} \mathrm{fb}$ directed spray of glyphosate @ $2000 \mathrm{~g} \mathrm{ha}^{-1}$ and pre emergence application of pendimethalin@1000 g ha ${ }^{-1} f b$ glyphosate @ $2000 \mathrm{~g} \mathrm{ha}^{-1}$ directed spray treatments.

This may be due to application of non selective herbicides at 45 and 60 DAS that might have resulted in reduced weed drymatter. Highest weed drymatter and the lowest weed control efficiency was observed at all the growth stages in weedy check treatment.

The lowest weed density was recorded in mechanical weeding thrice at 20,40 and 60 DAS across all the growth stages but it was on par with pre emergence application of pendimethalin@1000 $\mathrm{g} \mathrm{ha}^{-1} \mathrm{fb} 2 \mathrm{HW}$ at 20 and 50 DAS at 30 DAS and early post emergence application of pyrithiobacsodium @62.5 $\mathrm{g} \mathrm{ha}^{-1}+$ quizalofop- p- ethyl @ $50.0 \mathrm{~g} \mathrm{ha}^{-1} \mathrm{fb}$ manual weeding at 50 DAS and pre emergence application of pendimethalin@1000 g ha ${ }^{-1} f b$ glyphosate @ $2000 \mathrm{~g} \mathrm{ha}^{-1}$ directed spray treatments at 60 DAS, but it was significantly differed from all other treatments at 90 and 120 DAS, however it was followed by pre emergence application of pendimethalin @ $1000 \mathrm{~g} \mathrm{ha}^{-1} \mathrm{fb} 2 \mathrm{HW}$ at 20 and 50 DAS treatment but in turn this showed onpar weed density with all the treatments under study.
Lower weed density was observed in mechanical weeding thrice at 20,40 and 60 DAS treatment across all the growth stages except at 60 DAS, where lower weed density was observed in pre emergence application of pendimethalin @ $1000 \mathrm{~g} \mathrm{ha}^{-1}$ fb glyphosate@2000 g ha ${ }^{-1}$ directed spray treatment. This may be imposition of non selective herbicide treatment at 45 DAS (Table 2).

Plant height did not differ significantly at 30 DAS. However more plant height was observed with mechanical weeding thrice at 20, 40 and 60 DAS treatment and significantly superior over pyrithiobacsodium@62.5 g ha ${ }^{-1}, f b$ quizalofop- p- ethyl @ $50.0 \mathrm{~g} \mathrm{ha}^{-1}$, Pyrithiobac-sodium @ $62.5 \mathrm{~g}$ ha $^{-1}+$ quizalofop -p- ethyl @ $50.0 \mathrm{~g} \mathrm{ha}^{-1} \mathrm{fb}$ directed spray of paraquat @ $600 \mathrm{~g} \mathrm{ha}^{-1}$, Pyrithiobac -sodium @ $62.5 \mathrm{~g} \mathrm{ha}^{-1}+$ quizalofop - p- ethyl @ $50.0 \mathrm{~g} \mathrm{ha}^{-1} \mathrm{fb}$ directed spray of glyphosate @ $2000 \mathrm{~g} \mathrm{ha}^{-1}$, Pendimethalin@1000 $\mathrm{g} \mathrm{ha}^{-1} \mathrm{fb}$ glyphosate @ $2000 \mathrm{~g} \mathrm{ha}^{-1}$ directed spray and weedy check treatments. But it was comparable with remaining treatments at 60 DAS.

Significantly the lowest plant height was observed with weedy check, but remaining treatments showed onpar plant height with each other at 90 DAS.

But at 120 DAS significantly more plant height was observed with mechanical weeding thrice at 20,40 and 60 DAS and was onpar with pre emergence application of pendimethalin@ $1000 \mathrm{~g} \mathrm{ha}^{-1} \mathrm{fb} 2 \mathrm{HW}$ at 20 and 50 DAS and early post emergence application of pyrithiobac-sodium @ $62.5 \mathrm{~g}$ $\mathrm{ha}^{-1}+$ quizalofop- p- ethyl @ $50.0 \mathrm{~g} \mathrm{ha}^{-1} \mathrm{fb}$ manual weeding at 50 DAS treatments. The lowest plant height was observed with weedy check. 
More crop drymatter was noticed with mechanical weeding thrice at 20,40 and 60 DAS treatment at all growth stages and was onpar with pre emergence application of pendimethalin@1000 $\mathrm{g} \mathrm{ha}^{-1} \mathrm{fb} 2 \mathrm{HW}$ at 20 and 50 DAS treatment at 30 DAS, where as at $60 \mathrm{DAS}^{\mathrm{D}} \mathrm{T}_{9}$ treatment showed onpar crop drymatter production with $\mathrm{T}_{1}$ and $\mathrm{T}_{5}$ treatments.

However at 90 and 120 DAS mechanical weeding thrice at 20, 40 and 60 DAS significantly differed from all other treatments, but it was followed by pre emergence application of pendimethalin@ $1000 \mathrm{~g} \mathrm{ha}^{-1} \mathrm{fb} 2 \mathrm{HW}$ at 20 and 50 DAS treatment and inturn this was atpar with early post emergence application of pyrithiobac-sodium @ 62.5 g ha ${ }^{-1}+$ quizalofop- p- ethyl @ $50.0 \mathrm{~g} \mathrm{ha}^{-1} f b$ manual weeding at 50 DAS treatment. Significantly the lowest crop drymatter was observed with weedy check at all the growth stages (Table 3).

More number of boles per plant, kapas yield plant $^{+1}$ and kapas yield ha ${ }^{-1}$ was observed with mechanical weeding thrice at 20,40 and 60 DAS and was significantly differed from all other treatments under study, however it was followed by pre emergence application of pendimethalin $\mathrm{fb} 2 \mathrm{HW}$ at 20 and 50 DAS, inturn which was comparable with early post emergence application of pyrithiobac-sodium + quizalofop- - ethyl $f b$ manual weeding at 50 DAS. This result was reflected in terms of lower WI values of $0.01,15.3$ and 28.63 respectively (Table 4).

This might be due to lower weed drymatter, weed density, more crop drymatter and more number of boles plant ${ }^{-1}$. Similar findings were reported by Madhu et al. (2014) from Bt cotton at Bapatla.

Based on economic analysis of different weed management practices higher gross returns, net retuns and B.C ratio can be obtained from mechanical weeding thrice at 20, 40 and 60 DAS due to reduced cost of cultivation and increased yield. This inturn followed by pre emergence application of pendimethalin $\mathrm{fb} 2 \mathrm{HW}$ at 20 and 50 $\operatorname{DAS}\left(\mathrm{T}_{1}\right)$, inturn which was onpar with early post emergence application of pyrithiobac-sodium + quizalofop- p- ethyl $f b$ manual weeding $\left(\mathrm{T}_{5}\right)$ at $50 \mathrm{DAS}$.

It is concluded that, mechanical weeding thrice at 20, 40 and 60 DAS was found to be more economical to get higher kapass yield and net returns. However either pre emergence application of pendimethalin $\mathrm{fb}$ 2 hand weedings at 20 and 50 DAS or early post emergence application of pyrithiobacsodium@62.5 g ha ${ }^{-1}+$ quizalofop- p- ethyl quizalofop- p- ethyl @ $50.0 \mathrm{~g} \mathrm{ha}^{-1}$ at 20 DAS $f b$ manual weeding at 50 DAS was found to be economical with B.C ratio of 1.38 and 1.28 respectively.

\section{References}

Buchanan, G.A., and Burns, E.R. 1970. Influence of weed competition on cotton. Weed Sci. 18: 149-154.

Khadi, B.M., M.R.K, Rao and Singh, M. (2007). The Hindu survey of Indian agriculture 2007: Potential to improve lives of ryots. The Hindu.

Madhu, G., K. Srinivasulu, P. Prasuna Rani and Rao, A.S. 2014. Economics of rainfed $B t$ cotton as influenced by sequential application of herbicides. J. Cotton Res. Dev. 28 (2) 257-259.

Panwar, R.S and Malik, R.K.1991. Competition and control of weeds in cotton. J. Res. Haryana Agric. Univ. 21(3):226-234.

Pawar, A.K., B.M. Patil., A.P. Karunakar and Satao, R.N.2000. Effect of pre and post emergence herbicides on 
weed control and yield of cotton. Pestology. 24: 13-17.

Srinivasulu, G and Rao, A.S. 2000. Effect of sequential application of herbicides on weed management in cotton. In: Proceedings of symposium on challenges in Agronomic crop management in early 21st century organized by society of Agronomists, Hyderabad, May, 24-25. pp. 71-74.

\section{How to cite this article:}

Leela Rani, P., M. Yakadri and Ramprakash, T. 2016. Effect of Integrated Weed Management Practices on Growth and Yield of Bt-Cotton in Telangana State, India. Int.J.Curr.Microbiol.App.Sci. (2): 17-25.

doi: http://dx.doi.org/10.20546/ijcmas.2016.502.002 